\title{
Mathematical Modeling and Simulation of Ventricular Activation Sequences: Implications for Cardiac Resynchronization Therapy
}

\author{
Mark Potse
}

Received: 4 November 2011 / Accepted: 18 December 2011 / Published online: 27 January 2012

(C) The Author(s) 2012. This article is published with open access at Springerlink.com

\begin{abstract}
Next to clinical and experimental research, mathematical modeling plays a crucial role in medicine. Biomedical research takes place on many different levels, from molecules to the whole organism. Due to the complexity of biological systems, the interactions between components are often difficult or impossible to understand without the help of mathematical models. Mathematical models of cardiac electrophysiology have made a tremendous progress since the first numerical ECG simulations in the 1960s. This paper briefly reviews the development of this field and discusses some example cases where models have helped us forward, emphasizing applications that are relevant for the study of heart failure and cardiac resynchronization therapy.
\end{abstract}

Keywords Computer models •

Cardiac resynchronization therapy •

Electrocardiography $\cdot$ Cardiac electrophysiology

\section{Introduction}

Mathematical models play a prominent role in all natural sciences, from particle physics to medicine and

M. Potse ( $\square)$

Institute of Computational Science, University of Lugano,

Via Giuseppe Buffi 13, 6904 Lugano, Switzerland

e-mail:mark@potse.nl

\section{Potse}

Department of Biomedical Engineering,

Cardiovascular Research Institute Maastricht,

Maastricht University, Universiteitssingel 50,

6229 ER Maastricht, The Netherlands biology. Their role is complementary to that of observation. Whereas observations inform us about the behavior of nature itself, models inform us about the behavior of the theories that we use to express our understanding of nature. The purpose of a model is to translate a set of hypotheses (the theory) into predictions of observable events. These predictions can be compared to observations, and the underlying hypotheses can be rejected or corroborated. Mathematical models are required whenever such predictions cannot be made accurately enough by non-mathematical means. For all but the simplest cases, "mathematical model" is nowadays equivalent with "computer model." This paper overviews the contribution of computer models to our knowledge of cardiac electrophysiology and their potential to improve our understanding of heart failure and cardiac resynchronization therapy (CRT). The emphasis will be on ventricular and whole-heart models, and methodological aspects will be discussed only briefly, where necessary to explain the capabilities and limitations of models. Extensive reviews of the underlying methods have been published elsewhere $[14,58]$.

In Section "The Development of Computer Heart Models" of this paper, the development of computer heart models is outlined. In Section "Applications of Computer Heart Models", an overview is given of model applications, with an emphasis on heart failure and CRT.

\section{The Development of Computer Heart Models}

Membrane Models

The electrical activity of the heart originates in the ion channels, pumps, and exchangers in the membranes of 
myocytes and cells of the specific conduction system. The cells actively maintain a large difference in concentration of sodium, calcium, and potassium between their cytosol and the interstitium. Ion channels that are highly selective for one specific type of ion open and close at different transmembrane potentials, allowing ions to pass through the membrane along their electrochemical gradients. These ionic currents in turn affect the transmembrane potential, causing some channels to close and others to open. Hodgkin and Huxley captured the dynamics of the sodium and potassium currents of a neuron in mathematical equations and demonstrated that these give rise to action potentials [39]. Following this pioneering work, which was awarded with a Nobel prize in 1963, mathematical modeling of active cell membranes has been highly successful in testing whether the known mechanisms sufficed to account for all the experimentally observed phenomena [73]. Several detailed mathematical models for human cardiac myocytes have been published, both for atrial $[21,74]$ and for ventricular cells [44, 115]. Figure 1 shows schematically the components of a typical human ventricular membrane model [116].

Membrane models were in the first place developed in order to test the theoretical understanding of the mechanisms underlying the action potential. Another application of such models has been the prediction of drug effects on cardiac function [88]. Also, extensive studies have been performed to infer under what

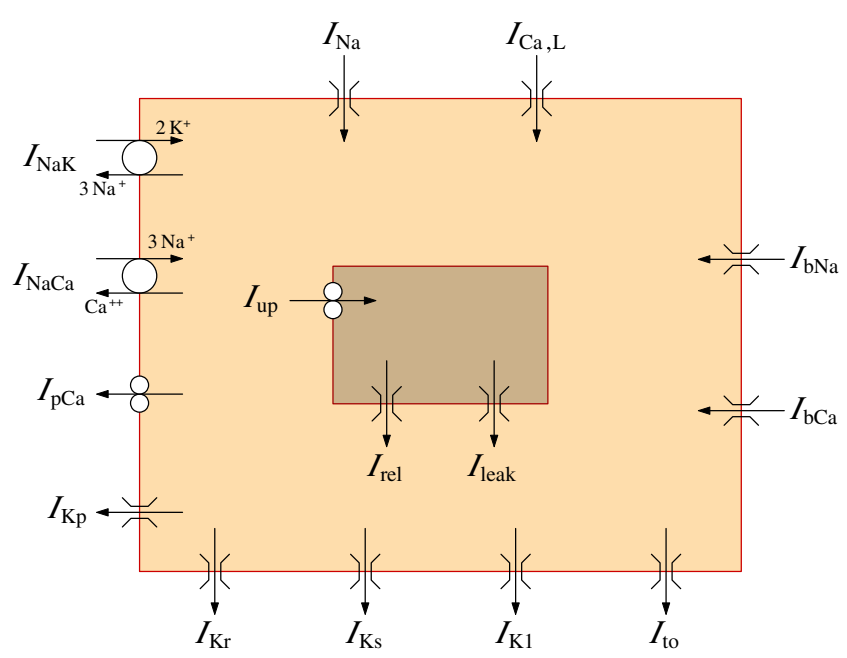

Fig. 1 Ion channels, pumps (double circles), and exchangers (large circles) in the model of the human ventricular myocyte published by Ten Tusscher et al. [116]. The large rectangle represents the cellular membrane; the small rectangle the sarcoplasmic reticulum (SR) membrane. The model keeps track of sodium, potassium, and calcium concentrations in the cytosol, as well as the calcium concentration in the SR circumstances a cell (model) would contribute to, or counteract abnormal automaticity $[9,57]$.

\section{Simulating Action Potential Propagation}

A membrane model represents the events in a single cell or, strictly speaking, in a small patch of membrane. This mimics the situation of an experimentally isolated cell or a patch clamp. Inside tissue, myocytes are coupled to each other by gap junctions [22], allowing the inward current in one cell to depolarize another cell and causing repolarization to be synchronized between cells [20]. By coupling membrane models together, it is possible to take these interactions into account and to create tissue models in which propagating activation can be simulated. With such models, it is possible to study not only the effect of drugs and mutations on cellular electrophysiology but also on action potential propagation and arrhythmia [18].

The first models of propagating action potentials, however, did not use this approach because it would have been too demanding for the computing equipment available at the time. Instead, simulations were based on the concept of cellular automata. Tissue models consisted of a number of "cells" (which did not necessarily have a one-to-one relationship with the biological cells) that could each be in one of a number of predefined states related to the excitable, absolute refractory, and relative refractory states of real myocardium.

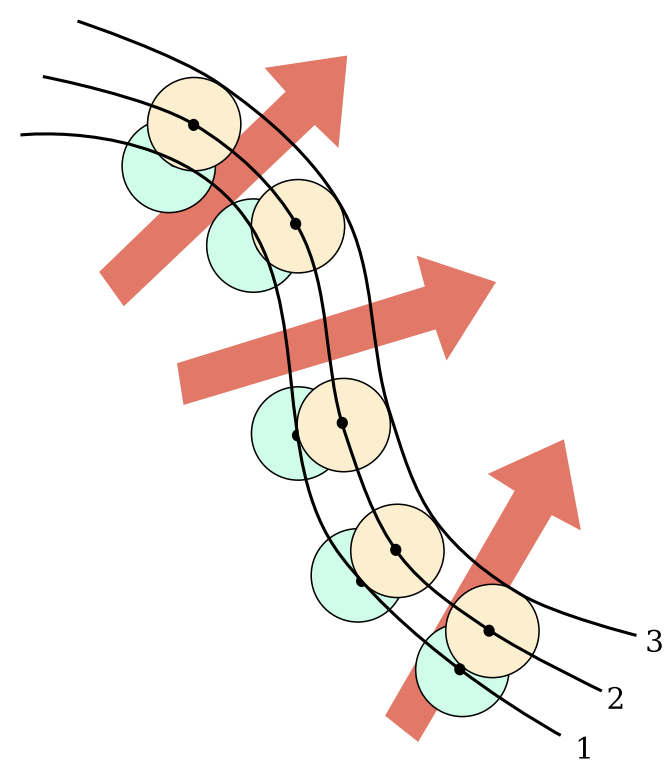

Fig. 2 Early heart models were based on Huygens' wavefront approach: Given an initial activation wavefront (which may be a single point or a surface), a sphere is placed on each point of the wavefront. The envelope of these spheres is the wavefront at the next time step, and the procedure is repeated 
Action potential propagation corresponded to a change from an excitable to a refractory state and was based on predetermined propagation velocities, using an approximation of Huygens' wavefront approach [43, 99] (Fig. 2).

Among the first papers reporting simulations of propagating action potentials are those by Moe et al. in 1964 [68], who simulated atrial fibrillation in a twodimensional sheet, and Okajima et al. in 1968 [76], who simulated activation in a three-dimensional model of the human ventricles. Solomon and Selvester [99] also simulated ventricular activation, using the early activation sites of the human ventricles published in 1970 by Durrer et al. [24] and comparing the simulated activation isochrones with those reported by Durrer.

\section{Computer Models of the ECG}

The earliest computer simulations of the ECG were published in the same period as the first simulations of propagating activation, but they did not yet make use of the latter [5, 30, 98]. Gelernter and Swihart in 1964 and Barr et al. in 1966 published methods to compute the potential distribution on the surface of an inhomogeneous torso model that would result from a given configuration of oriented current sources ("dipoles") in the heart $[5,30]$. These dipoles were configured by hand [98], or derived from an assumed action potential amplitude and measured activation wavefronts [31].

Shortly after the first publications of electrocardiographic body surface maps [107], Gelernter et al. [31] reported simulated electrocardiographic body surface maps based on measured activation wavefronts in canine hearts provided by Scher and coworkers [36, 96]. Subsequently, Selvester et al. [98] presented complete QRS complexes, also based on measured activation patterns.

Miller and Geselowitz in 1978 computed the QRS complex and $\mathrm{T}$ wave in the 12-lead ECG [64] based on activation sequences computed earlier by Solomon and Selvester [99]. A similar model was used by Gulrajani and Mailloux to quantify the effect of the lungs and intracavitary blood masses on the ECG [35].

Integration of simulated propagation and ECG computation was reported in a few studies in the 1970s, for example those by Salu and Rush [93] and by Niimi et al. [72]. In the 1980s, several groups reported detailed models of the heart and torso in which both propagation and electric potentials were simulated [2, 26, 59]. A new development was the use of fiber orientations (based on the analysis of Streeter et al. [104]) so that anisotropic propagation velocities could be used $[59,60]$. ECGs were simulated with detailed, inhomo- geneous torso models that included the lungs and the skeletal muscle layer. Another novelty was that these models assumed complete action potential waveforms rather than only upstrokes, so that also $\mathrm{T}$ waves could be simulated. However, propagation velocities and action potentials were still predetermined, not simulated.

\section{The Bidomain and Monodomain Models}

An important breakthrough occurred with the development of the bidomain model of cardiac muscle $[64,97,114]$, a conceptual model that allows a description of electrical current flow in the tissue. The actual structure of myocytes connected to each other by gap junctions, forming branching and intertwining muscle fibers embedded in an interstitial fluid and interspersed with capillaries and other structures, is abstracted by two continuous domains. These are the intracellular domain, representing the myocytes and gap junctions, and the extracellular domain, representing all other

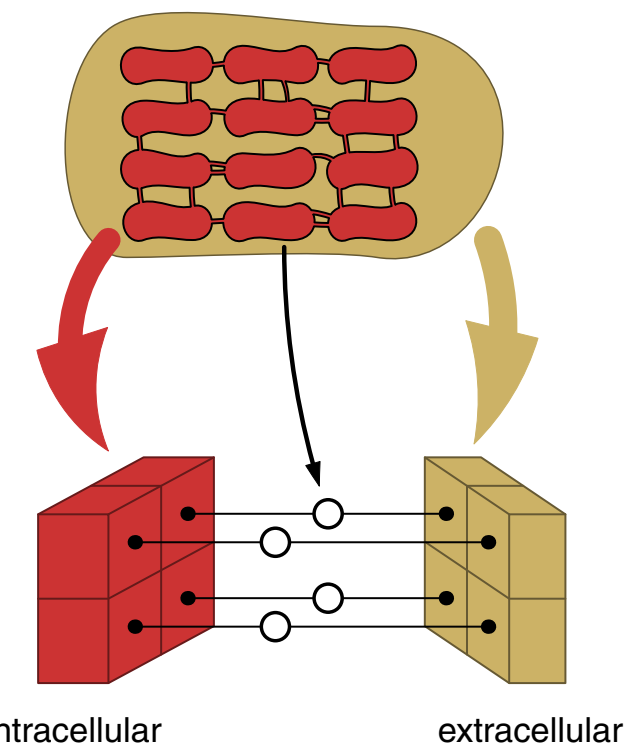

Fig. 3 In a bidomain model of the heart, the myocardium is abstracted as two continuous media separated by the cell membrane. One medium represents the intracellular domain consisting of cells and gap junctions, and the other represents the extracellular domain. These media are electrically conducting to represent the conductance of gap junctions and extracellular fluid, respectively. The conductivity is inhomogeneously anisotropic to represent the varying fiber directions in the heart. The two media are then split into small blocks, the sizes of which are dictated primarily by the properties of the mathematical equations that govern the potential fields. Each block is connected to the corresponding block in the other domain by a membrane model, which computes the transmembrane current flowing from one domain to the other as a function of time and potential difference 
tissue components. Each domain is characterized by an electrical conductivity that is greater along than across the fibers and changes from place to place, to account for the variable fiber orientation in the heart. The two domains are electrically coupled only through the cell membrane, as illustrated in Fig 3.

The idea to treat the cardiac tissue as a continuum (while acknowledging that it is in reality composed of discrete cells with membranes) is often attributed to Otto Schmitt, who gave a qualitative description of it in 1969 [97]. The mathematical expression of the bidomain was published independently by Miller and Geselowitz [64] and by Tung [114] in 1978.

The bidomain theory had three important consequences. First, it gave a firm theoretical ground to the current dipoles that had been used for decades to represent the cardiac activity [59]. Second, because the bidomain distinguishes intracellular and extracellular current flows, it became possible to simulate the application of external stimuli or defibrillation currents [69]. Third, the bidomain led to a completely new type of heart model that was built on the membrane models discussed in Section "Membrane Models". These "reaction-diffusion models" will be discussed in Section "Reaction-Diffusion Models".

An important simplification of the bidomain model results when the electrical resistance is assumed to reside completely in the intracellular domain (Fig 3). This assumption prohibits simulation of extracellular potential fields because the zero resistance in the extracellular domain implies a uniform potential there. However, it has a negligible effect on propagating activity in a reaction-diffusion model $[8,80]$, and it allows for much more efficient simulations. Models that use this assumption are termed monodomain models.

\section{Reaction-Diffusion Models}

In the 1990s, it became feasible to couple enough membrane models together to form a complete pair of ventricles, or even a complete heart. This coupling was achieved through the monodomain or bidomain model, which approximate the myocardium by one or two continuous domains, respectively. This is a necessary step to make calculations on the scale of a whole heart feasible. Even today, dealing with each of the heart's two billion myocytes individually is not within reach. Fortunately, we can be confident that at least in structurally normal tissue this simplification does little harm. There exist mathematical models that do incorporate the microstructure of the heart $[45,102]$, but they are presently limited to preparations of at most a few millimeters length.
Even when a model assumes a continuous (bi)domain, it is necessary for numerical simulation to divide the domain into discrete elements. The steepness of the membrane potential upstroke and the resulting steep spatial potential gradients dictate an element size of at most 0.25 to $0.5 \mathrm{~mm}$ (depending on other methodological aspects). Consequently, published models of the human heart had in the order of 10 million to 100 million elements [7, 40,71, 113].

With this type of model, the assumptions of predetermined propagation velocities and predetermined action potential waveforms could be abandoned. Propagation and action potentials could be computed simultaneously with the membrane ionic currents. Changes could be made at the level of these currents, and the effects on propagating activation, electrograms, and the ECG could be simulated, as illustrated in Fig 4. Similarly, pacing or defibrillating currents could be injected in the models, and their effects on the ionic currents could be studied [111].

With the new possibilities came new challenges. In order to obtain $\mathrm{T}$ waves that have the same sign as the QRS complex in the same lead, a characteristic known as T-wave concordance and observed in nearly all leads of the normal human 12-lead ECG, dispersion

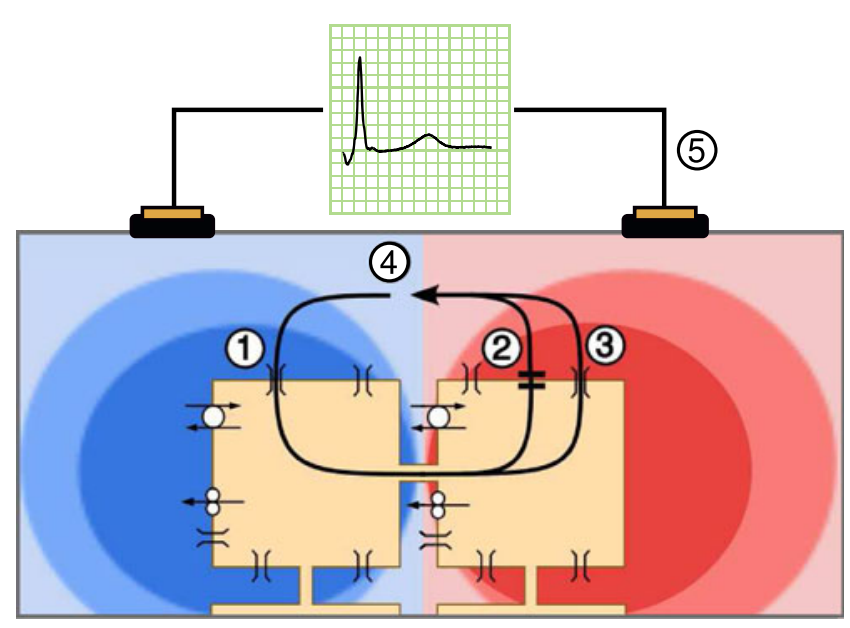

Fig. 4 Principle underlying membrane-based heart models. Two model cells are depicted schematically, with their ion channels, pumps, and exchangers. Gap junctions connect the cells. In depolarizing cells, a large inward sodium current flows along its electrochemical gradient (1). This current passes through gap junctions to neighboring cells. There, part of it serves to charge the cell membrane (2) represented by a capacitor symbol, and another part leaks through potassium channels that are still open (3). The two parts form an outward current in this cell, and the current loop is closed in the interstitium and outside the heart (4), where it generates a potential field, schematically indicated here in red for positive potentials and blue for negative potentials. This potential field is picked up as an ECG (5) 
of action potential duration is necessary [79]. On average, late-activated cells should repolarize earlier than early-activated cells. This requires a strong negative correlation between activation time and action potential duration. Previously, this heterogeneity had been included in the models by directly manipulating the action potential waveforms in different regions. In membrane-based models, heterogeneity of action potential duration can only be obtained from heterogeneity of ion-channel densities or characteristics. Attempts to reproduce realistic $\mathrm{T}$ waves based on known heterogeneities of ion channels have shown that these have still not been sufficiently characterized to explain concordant $\mathrm{T}$ waves in the human ECG $[19,109,116]$. Because of the large uncertainty in several parameters, such as the regional expression level of the slow delayed rectifier current $\left(I_{\mathrm{Ks}}\right)$, it is common practice to assume a transmural and/or apicobasal gradient in $I_{\mathrm{Ks}}$ density to obtain concordant $T$ waves [52].

\section{Eikonal Models}

While membrane-based heart models are much more realistic than the earlier fixed-velocity models, they are computationally much more expensive. For problems where only the activation wavefront is of interest and its velocities along and across the fibers can be assumed constant, an elegant solution is possible by the use of an "eikonal equation" [15, 49]. Like Huygens' approach, on which earlier models were based, this type of equation originates from the theory of optics [48]. Compared to approximations of Huygens' approach, an eikonal equation is more difficult to solve, but also more accurate.

\section{Electromechanical Models}

In order to study the effect of modified cardiac activation on cardiac pump function, it is necessary to construct models that incorporate not only the electrophysiology but also the electromechanical coupling, mechanical contraction, mechanoelectric feedback, and even the fluid dynamics and hemodynamic feedback on the electrical and mechanical function of the heart. Such models are vastly more complicated than models of electrophysiology alone and contain many more free parameters. Several groups have presented models that incorporated at least some of these aspects $[1,50,54$, $55,106]$. Recent reviews were published by Williams et al. [123], Trayanova et al. [112], and by Kuijpers et al. (this issue) [56]. This paper will concentrate on purely electrophysiological models.

\section{Applications of Computer Heart Models}

\author{
Model Studies of Cardiac Arrhythmia
} and Defibrillation

Many modeling studies have addressed the initiation and termination of arrhythmia. A complete account of these is outside the scope of this review; only some examples will be discussed here. Several studies have addressed the role of specific ionic currents in arrhythmia. For example, Bernus et al. considered the role of the L-type Ca current in ventricular fibrillation [7]. Detailed membrane models made it possible to study the effect of genetic mutations affecting specific ion channels on the function of the cell and on the initiation and maintenance of arrhythmia in the whole heart [13, 34, 40, 51, 94, 121]. Several groups studied the role of the Purkinje system in polymorphic ventricular tachycardia $[6,10]$. On a more fundamental level, the basic principles of reentry [17] and unidirectional block [27] have been elucidated with computer models. Model studies have contributed importantly to our understanding of virtual electrodes, the phenomenon that activation of tissue can occur at a distance from a stimulating electrode rather than directly underneath it $[16,25,122]$. Not only the initiation but also the termination of arrhythmia has received attention with many studies investigating factors in defibrillation success $[86,89,111]$.

\section{Linking Signal Shapes to Underlying Phenomena}

An important role of models is to give insight in the relation between observable signals and underlying quantities that are difficult or impossible to observe. Cardiac electrograms (measured with electrodes that touch the myocardium) are much easier to record than the transmembrane potentials, which we are actually interested in. The relation between the two is not straightforward because the extracellular potentials that the electrogram records depend not only on the local transmembrane potential but on all transmembrane potentials in the heart [83]. Still, with a careful analysis, much information can be derived from these signals. Using both experiments and mathematical models, Spach and Barr explained the relation between the rapid downstroke in the unipolar electrogram and the onset of the action potential in nearby tissue $[100,101]$. Several other aspects of the unipolar electrogram, including complex R waves [38, 108] and the relation between $\mathrm{T}$-wave parameters and local repolarization [83, 95], were subsequently clarified with computer models. 
The monophasic action potential (MAP) [28] is another example of a signal that has a nontrivial relation to the action potential waveform. To make a MAP recording, the tissue is locally depolarized by applying pressure. As a result, the local extracellular potential will contain a large contribution from the intracellular potentials of the tissue just outside the depolarized area. A reference potential is obtained from an electrode a few millimeters away into the cavity. The difference between these potentials yields a signal that resembles an action potential, a signal that is normally measured between the intracellular and extracellular potentials at the same location. The precise mechanism of the depolarization and the degree to which the cells depolarize are uncertain, but the physics behind the MAP are well understood, and its working has been illustrated in several computer model studies $[110,119,120]$.

The effect of changes in ion-channel function on the surface ECG is often difficult to predict. Modeling studies of a one-dimensional preparation reflecting the transmural heterogeneity in the ventricles have demonstrated several of these effects on endocardialto-epicardial pseudo-ECGs that could be compared to laboratory measurements in wedge preparations [33]. More recently, it has become feasible to extend such predictions to the full 12-lead ECG generated by the in situ heart. As an example, Hoogendijk et al. have shown that reduced sodium current, as occurs in Brugada syndrome, by itself cannot explain the Brugada sign in the ECG; only when it was combined with muscle damage was right-precordial ST-segment

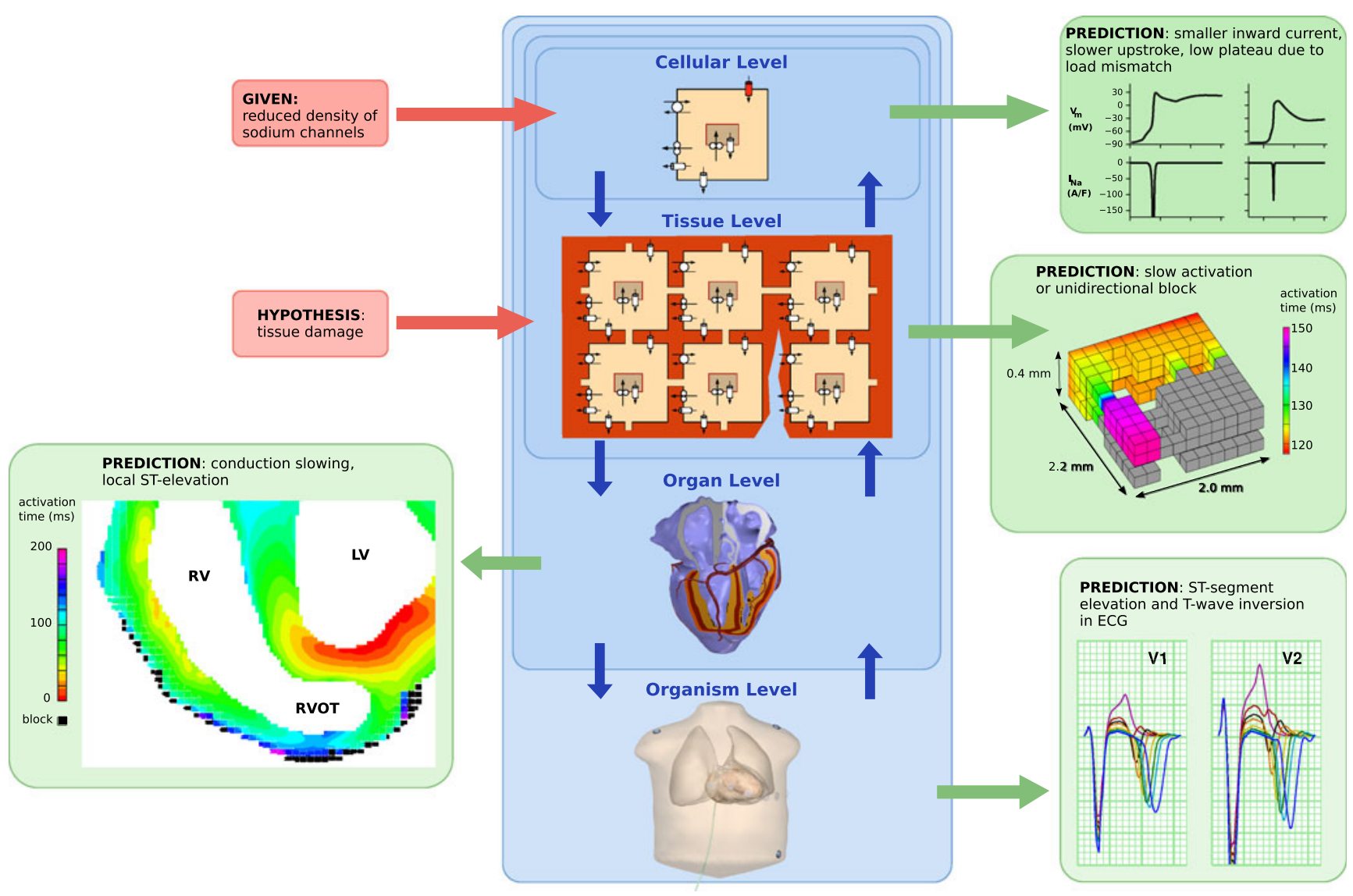

Fig. 5 Example of a computer model study to link events at the organ and organism levels to pathologies on the cellular and tissue levels. The model (blue) was a reaction-diffusion model of the heart, consisting of millions of membrane models, coupled to a torso model. On the cellular level, the sodium channel density was reduced in the entire heart. On the tissue level, heterogeneous uncoupling was assumed. Model predictions were obtained on all levels. On the cellular level, the model predicted reduced inward current and slower action potential upstrokes, as expected. On the tissue level, conduction slowing and block was observed at sites where narrow strands of myocardium had to activate larger volumes. On the organ level, this resulted in areas of conduction slowing and block in the subepicardium. On the organism level, ST-segment elevation and T-wave inversion were predicted that could be modulated by changing the expression of L-type calcium current (different colors of traces). Parts of this figure were reprinted from Hoogendijk et al. [40, 41] with permission from Elsevier 
elevation reproduced [40, 41]. In these studies, tissuelevel and organ-level results were obtained from experiments in tissue samples and an isolated human heart, respectively. A large-scale computer model of the heart and torso was used to evaluate the corresponding ECG features and to clarify the events at the cellular, tissue, and organ levels, as illustrated in Fig 5.

\section{Ischemic Heart Disease}

Acute myocardial ischemia that affects the full thickness of the ventricular muscle is known to cause ST-segment elevation in overlying electrocardiographic leads. This is often accompanied by "reciprocal" ST depression on the other side of the thorax. Although this has often been explained as a necessary consequence of the primary ST elevation, several authors have suggested that "ischemia at a distance," that is, a lesser degree of ischemia remote from the primary zone, would be involved $[12,75]$. A simulation study by Dubé et al. in 1996 showed convincingly that such a hypothesis is not necessary [23]. Assuming only a primary ischemic zone, they were able to reproduce the surface patterns of ST elevation and depression that could be observed after temporary occlusions of the coronary arteries during angioplasty. Another observation from the same study was that the somewhat counterintuitive shortening of the QRS complex during ischemia could well result from slower conduction in the bundle branches; changes in cancelation then caused QRS shortening in some leads [23].

In case of subendocardial ischemia, textbooks still promote the idea that overlying electrocardiographic leads will show ST depression. Recent studies with computer models have shown that more requirements have to be fulfilled before subendocardial ischemia can lead to measurable ST depression [42, 47, 81]. These studies suggest that primary ST depression (ST depression in the absence of ST elevation) observed in patients indicates a global perfusion problem rather than a local one. This may explain the observation that the location of primary ST depression cannot identify the obstructed artery in patients undergoing coronary angioplasty [70].

\section{Heart Failure, Conduction Disturbances, and CRT}

In patients with left ventricular dysfunction and in patients with a right-ventricular pacemaker, discoordinate contraction of the left ventricular septum and free wall reduces the pumping efficiency of the heart $[54,105]$. The purpose of CRT is to resynchronize the mechanical contraction of the ventricular wall. Evidently, a rapid electrical activation is a prerequisite for synchronous contraction. An important challenge in CRT is to find the best pacing locations and atrioventricular and interventricular delays. The solution likely depends on the individual anatomy and pathophysiology of the patient's heart. An important body of work has therefore been dedicated to the construction of patient-tailored models in which the importance of pathology, geometry, and CRT tuning may be tested. For example, Miri et al. $[65,87]$ computed biventricular-paced activation sequences and ECGs in ten patient-specific heart models to simulate the process of CRT tuning. They found that in addition to AV and VV delays, the position of the LV electrode influenced electrical synchrony and that the optimal position was patient-dependent.

Retrograde activation of the Purkinje system may well contribute to the success of individual CRT treatments. It is still uncertain whether activation does reenter the Purkinje system in left bundle-branch block (LBBB) or biventricular pacing. Lorange et al. simulated LBBB with and without the possibility of reentry and found that the simulated ECG and epicardial isochrones in the situation with reentry provided the best agreement with experimental data [61]. Romero et al. simulated biventricular pacing and found that, when retrograde Purkinje activation was permitted, the endocardial activation sequence was more realistic [90]. Kerckhoffs et al. [53] argued for the existence of a rapidly conducting subendocardial layer in addition to the Purkinje system. They found that, without retrograde Purkinje activation, assuming a 1.7 times faster conduction in the subendocardial layer helps to obtain realistic epicardial activation patterns during LV pacing.

Although Lorange et al. reported that retrograde Purkinje activation allowed for more realistic simulation results in case of LBBB, they also found that to obtain typical patterns of left anterior fascicular block (LAFB) [91], it was necessary not only to cut off a part of the LV Purkinje network but also to disable reentry in the disconnected part [61]. Bacharova et al. recently reported that the ECG pattern of LAFB can also be caused by reduced coupling in the myocardium, without damage to the specific conduction system [4]. Figure 6 shows simulated ECGs and vectorcardiograms in the case of "true" LBBB (interruption of the left bundle branch) and without LBBB but with $60 \%$ uncoupling in the LV myocardium, using a reaction-diffusion model of the human heart and torso [4, 82]. The QRS angles in the two cases are comparable, but there is a large difference in amplitude and QRS duration.

Most patients with LBBB ECGs do not have large amplitudes like those shown in Fig 6. It is presently 

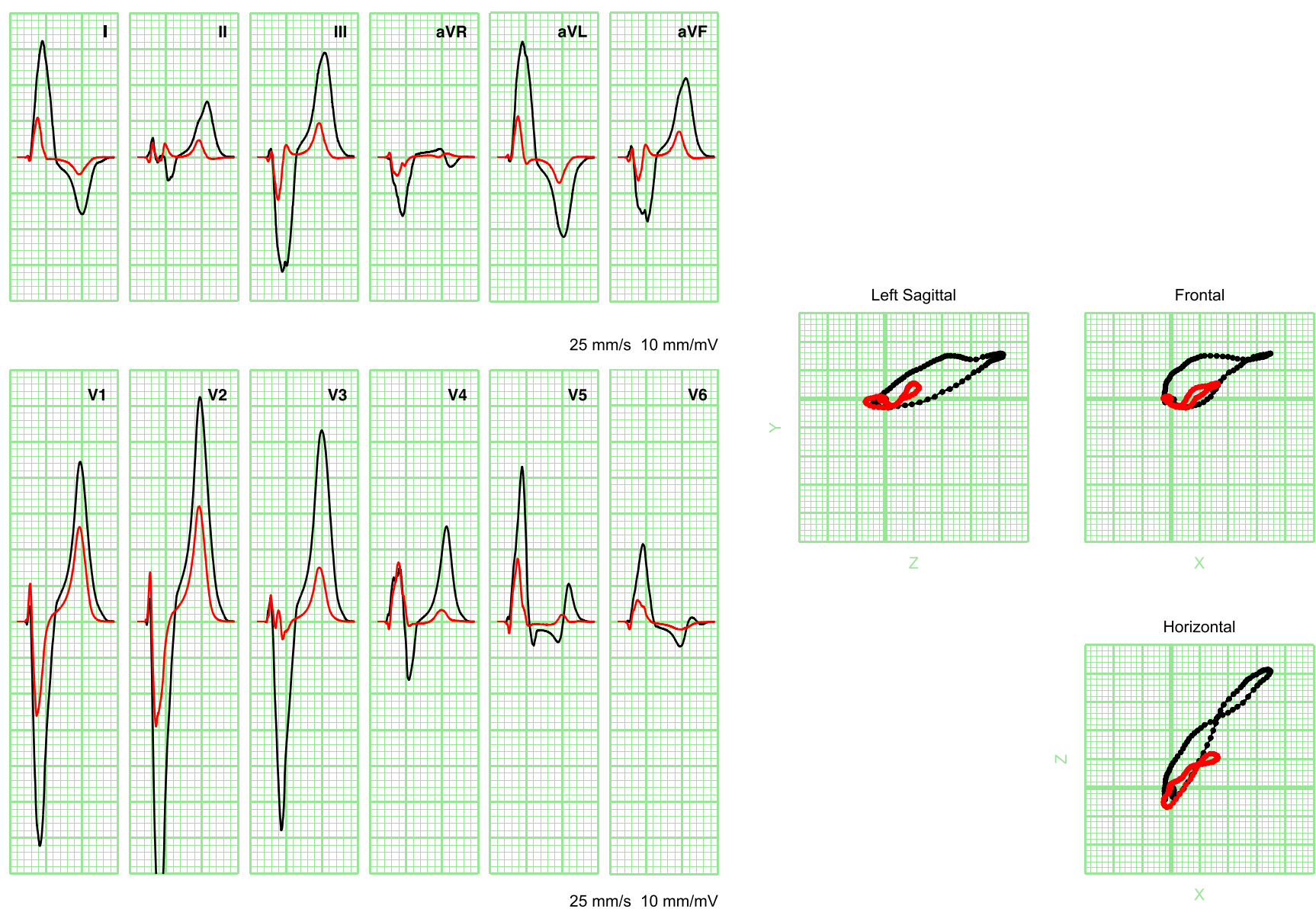

$25 \mathrm{~mm} / \mathrm{s} 10 \mathrm{~mm} / \mathrm{mV}$

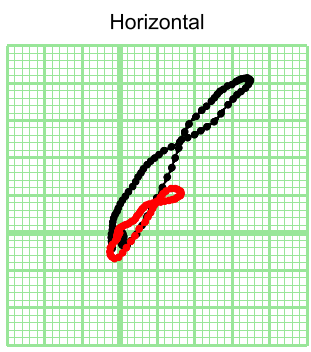

Fig. 6 Simulated ECG and vectorcardiograms with true LBBB (black) and without LBBB but with $60 \%$ uncoupling in the LV myocardium (red)

not clear whether this indicates that these patients do not actually have a dysfunctioning bundle branch, or whether they simply have other pathologies that reduce QRS amplitude, counteracting the lack of electrocardiographic cancelation in the LV that allows the QRS to become so large in the model. Strauss et al. recently argued that in case of "true" LBBB, the QRS duration must be well over $140 \mathrm{~ms}$ [103]. The model results suggest that in other cases, LBBB and LBBB-like ECG patterns may be due to uncoupling in the working myocardium. Alternatively, remodeling of membrane ionic currents could contribute to such ECG patterns; a hypothesis that has not yet been evaluated with a numerical model.

Hypertrophy and dilatation are common factors in ventricular dysfunction. To explain the electrocardiographic changes seen in patients with left ventricular hypertrophy or dilatation, the Brody effect [11] has often been quoted. The Brody effect is the amplification of radial current sources due to the rel- atively high electrical conductivity of the intracavitary blood. When the cavity size increases, the amplitude of the QRS complex-which is mostly due to radial sources-should also increase. One of the earliest applications of mathematical modeling to better understand the ECG was the 1966 paper by Gelernter et al. [31] in which they quantified the Brody effect (apparently unaware of Brody's publication [11] 10 years earlier) for the case of a thickened ventricular muscle mass, with or without enlarged cavity. Their purpose was to investigate the hypothesis that these geometrical changes could explain the unpublished observation by A. M. Scher that increasing heart size in training marines was accompanied by decreasing QRS amplitude. As we would expect based on Brody's more qualitative argument, they found that this was not the case. More recent numerical studies evaluating the Brody effect confirmed its existence but cautioned that its effects are not always straightforward $[35,78,92]$. 


\section{Inverse Models}

So far, only so-called forward models have been discussed, that is, models that simulate events based on hypotheses. Inverse models or "electrocardiographic imaging" methods, in contrast, take measured signals as input and attempt to reconstruct the underlying events [32, 37, 63, 66, 85]. The term "imaging" should be interpreted with care. On the one hand, the name is appropriate because, similar to other medical imaging methods such as computed tomography (CT) and magnetic resonance imaging (MRI), electrocardiographic imaging reconstructs invisible events from measured signals. On the other hand, it should be remembered that electrocardiographic imaging is much more uncertain than MRI or CT. Surface signals only provide a very brief summary of the activity of the heart's two billion myocytes. For each set of measured data, there is an infinite number of (physically) possible source configurations [62]. Therefore, inverse models rely on many assumptions in order to arrive at a unique answer. Commonly made assumptions are that the tissue is homogeneous and has isotropic conductivity [67]. When in reality this is not the case, the inverse model may report an incorrect activation order rather than reporting an inhomogeneity.

Nevertheless, inverse models can give intriguing results which, when interpreted judiciously, may point us to important findings. Such is the case for the observation that noncontact electrograms in LBBB patients indicate a U-shaped activation pattern around a functional line of block $[3,29]$. This line of block was not confirmed by bipolar contact electrograms in the same patients [3]. This discrepancy was interpreted as indicating that the subendocardial activation pattern differs from that in deeper layers. An alternative interpretation is that the lateral wall in these patients, two thirds of whom had been diagnosed with idiopathic cardiomyopathy [3], suffered more cellular uncoupling than the septum. This would lead to an area of slow conduction and fractionated low-amplitude electrograms. One can imagine that the mapping system, unable to recognize such a heterogeneity in passive tissue properties and forced to explain the measured signals in terms of propagating activation wavefronts, would come up with the reported $U$ shape. When the ventricles were paced from the region where block had occurred, no signs of block were found on non-contact mapping [3]. This finding also can be explained in two ways. Either the block was functional rather than anatomical or the solution algorithm was better able to recognize activation in this area when it was not masked by activation elsewhere.
Interestingly, apparent lines of block in the lateral wall were also reported in reconstructed epicardial activation patterns based on body-surface mapping [46]. Whether this confirms the findings from endocardial noncontact mapping or suffers from the same problem (a low-amplitude region interpreted as a region of block) is presently not clear. Several other interesting hints about the activation patterns in heart failure patients and CRT recipients resulted from this study and others from the same group [46, 117, 118].

\section{Conclusion}

Heart failure is a complex disease that involves remodeling at the level of the whole organ, the tissue, and the membrane ionic currents. At each of these levels, there is an effect on the ECG. Without the help of numerical models, it would be difficult to attribute observed changes in ECG waveforms to individual causes. One example has been mentioned above: Reduced tissue conductivity due to cardiomyopathy can mimic the ECG pattern of left anterior fascicular block and at the same time mask the ECG signs of left ventricular hypertrophy by reducing QRS amplitude [4]. So if, after biventricular pacemaker implantation for CRT, increasing QRS amplitude is observed, one could wonder whether this is a good sign indicating reversal of cardiomyopathy or a bad sign indicating ongoing dilatation. By careful comparisons of simulated and measured ECGs, we may be able to learn how to distinguish the two, or we may find that the ECG, crucial as it is to diagnose electrophysiological remodeling, simply cannot provide reliable information about anatomical remodeling.

In this and other domains of cardiac electrophysiology, computer models have a unique role next to clinical and experimental research. Models translate between unobservable and observable variables, serve as a training tool $[77,84]$, and, in the case of inverse models, suggest explanations for observed phenomena.

Open Access This article is distributed under the terms of the Creative Commons Attribution License which permits any use, distribution, and reproduction in any medium, provided the original author(s) and the source are credited.

\section{References}

1. Aguado-Sierra, J., Krishnamurthy, A., Villongco, C., Chuang, J., Howard, E., Gonzales, M. J., et al. (2011). 
Patient-specific modeling of dyssynchronous heart failure: A case study. Progress in Biophysics and Molecular Biology, 107, 147-155. doi:10.1016/j.pbiomolbio.2011.06.014.

2. Aoki, M., Okamoto, Y., Musha, T., \& Harumi, K. I. (1987). Three-dimensional simulation of the ventricular depolarization and repolarization processes and body surface potentials: Normal heart and bundle branch block. IEEE Transactions on Biomedical Engineering, 34(6), 454-462.

3. Auricchio, A., Fantoni, C., Regoli, F., Carbucicchio, C., Goette, A., Geller, C., et al. (2004). Characterization of left ventricular activation in patients with heart failure and left bundle-branch block. Circulation, 109, 1133-1139.

4. Bacharova, L., Mateasik, A., Krause, R., Prinzen, F., Auricchio, A., \& Potse, M. (2011). The effect of reduced intercellular coupling on electrocardiographic signs of left ventricular hypertrophy. Journal of Electrocardiology, 44, 571-576. doi:10.1016/j.jelectrocard.2011.06.004.

5. Barr, R. C., Pilkington, T. C., Boineau, J. P., \& Spach, M. S. (1966). Determining surface potentials from current dipoles, with application to electrocardiography. IEEE Transactions on Biomedical Engineering, 13(2), 88-92.

6. Berenfeld, O., \& Jalife, J. (1998). Purkinje-muscle reentry as a mechanism of polymorphic ventricular arrhythmias in a 3 -dimensional model of the ventricles. Circulation Research, 82, 1063-1077.

7. Bernus, O., van Eyck, B., Verschelde, H., \& Panfilov, A. V. (2002). Transition from ventricular fibrillation to ventricular tachycardia: A simulation study on the role of $\mathrm{Ca}^{2+}$-channel blockers in human ventricular tissue. Physics in Medicine \& Biology, 47, 4167-4179.

8. Bishop, M. J., \& Plank, G. (2011). Representing cardiac bidomain bath-loading effects by an augmented monodomain approach: Application to complex ventricular models. IEEE Transactions on Biomedical Engineering, 58, 1066-1075.

9. Bouchard, S., Jacquemet, V., \& Vinet, A. (2011). Automaticity in acute ischemia: Bifurcation analysis of a human ventricular model. Physical Review E, 83, 011,911. doi:10.1103/PhysRevE.83.011911.

10. Boyle, P. M., Deo, M., Plank, G., \& Vigmond, E. J. (2010). Purkinje-mediated effects in the response of quiescent ventricles to defibrillation shocks. Annals of Biomedical Engineering, 38, 456-468.

11. Brody, D. A. (1956). A theoretical analysis of intracavitary blood mass influence on the heart-lead relationship. Circulation Research, 4, 731-738.

12. Brymer, J. F., Khaja, F., Marzilli, M., Goldstein, S., \& Alban, J. (1985). "Ischemia at a distance" during intermittent coronary artery occlusion: A coronary anatomic explanation. Journal of the American College of Cardiology, 6(1), 41-45.

13. Clancy, C. E., \& Rudy, Y. (2002). $\mathrm{Na}^{+}$channel mutation that causes both Brugada and long-QT syndrome phenotypes; a simulation study of mechanism. Circulation, 105, 1208-1213.

14. Clayton, R. H., Bernus, O., Cherry, E. M., Dierckx, H., Fenton, F. H., Mirabella, L., et al. (2011). Models of cardiac tissue electrophysiology: Progress, challenges and open questions. Progress in Biophysics and Molecular Biology, 104, 22-48 (review).

15. Colli Franzone, P., Guerri, L., \& Tentoni, S. (1990). Mathematical modeling of the excitation process in myocardial tissue: Influence of fiber rotation on wavefront propagation and potential field. Mathematical Biosciences, 101(2), 155235.

16. Colli-Franzone, P., Pavarino, L. F., \& Scacchi, S. (2011). Exploring anodal and cathodal make and break cardiac ex- citation mechanisms in a 3D anisotropic bidomain model. Mathematical Biosciences, 230, 96-114.

17. Comtois, P., \& Vinet, A. (2002). Resetting and annihilation of reentrant activity in a model of a one-dimensional loop of ventricular tissue. Chaos, 12(3), 903-922.

18. Comtois, P., Sakabe, M., Vigmond, E. J., Munoz, M., Texier, A., Shiroshita-Takeshita, A., et al. (2008). Mechanisms of atrial fibrillation termination by rapidly unbinding $\mathrm{Na}^{+}$channel blockers: Insights from mathematical models and experimental correlates. American Journal of Physiology. Heart and Circulatory Physiology, 295, H1489-H1504.

19. Conrath, C. E., \& Opthof, T. (2006). Ventricular repolarization: An overview of (patho)physiology, sympathetic effects and genetic aspects. Progress in Biophysics and Molecular Biology, 92(3), 269-307.

20. Conrath, C. E., Wilders, R., Coronel, R., de Bakker, J. M., Taggart, P., de Groot, J. R., et al. (2004). Intercellular coupling through gap junctions masks $\mathrm{M}$ cells in the human heart. Cardiovascular Research, 62, 407-414.

21. Courtemanche, M., Ramirez, R., \& Nattel, S. (1998). Ionic mechanisms underlying human atrial action potential properties: Insights from a mathematical model. American Journal of Physiology. Heart and Circulatory Physiology, 275, H301-H321.

22. Desplantez, T., Dupont, E., Severs, N. J., \& Weingart, R. (2007). Gap junction channels and cardiac impulse propagation. The Journal of Membrane Biology, 218, 13-28 (review).

23. Dubé, B., Gulrajani, R. M., Lorange, M., LeBlanc, A. R., Nasmith, J., Nadeau, R. A. (1996). A computer heart model incorporating anisotropic propagation: IV. Simulation of regional myocardial ischemia. Journal of Electrocardiology, 29(2), 91-103.

24. Durrer, D., van Dam, R. T., Freud, G. E., Janse, M. J., Meijler, F. L., \& Arzbaecher, R. C. (1970). Total excitation of the isolated human heart. Circulation, 41(6), 899-912.

25. Efimov, I. R., Gray, R. A., \& Roth, B. J. (2000). Virtual electrodes and deexcitation: New insights into fibrillation induction and defibrillation. Journal of Cardiovascular Electrophysiology 11, 339-353.

26. Eifler, W. J., Macchi, E., Ritsema van Eck, H. J., Horacek, B. M., \& Rautaharju, P. M. (1981). Mechanism of generation of body surface electrocardiographic P-waves in normal, middle, and lower sinus rhythms. Circulation Research, 48, 168-182.

27. Fast, V. G., \& Kléber, A. G. (1995). Block of impulse propagation at an abrupt tissue expansion: Evaluation of the critical strand diameter in 2- and 3-dimensional computer models. Cardiovascular Research, 30, 449-459.

28. Franz, M. R., Bargheer, K., Rafflenbeul, W., Haverich, A., \& Pichtlen, P. R. (1987). Monophasic action potential mapping in human subjects with normal electrocardiograms: Direct evidence for the genesis of the $\mathrm{T}$ wave. Circulation, 75, 379-386.

29. Fung, J. W. H., Yu, C. M., Yip, G., Zhang, Y., Chan, H., Kum, C. C., et al. (2004). Variable left ventricular activation pattern in patients with heart failure and left bundle branch block. Heart, 90, 17-19.

30. Gelernter, H. L., \& Swihart, J. C. (1964). A mathematicalphysical model of the genesis of the electrocardiogram. Biophysical Journal, 4, 285-301.

31. Gelernter, H. L., Swihart, J. C., \& Angell, M. A. K. (1966). The use of a mathematical model in the study of the properties of the full-surface electrocardiogram. I: Scher generators in a homogeneous torso. II: "Enlarged heart" potentials. Annals of the New York Academy of Sciences, 28, 1069-1084. 
32. Ghosh, S., Cooper, D. H., Vijayakumar, R., Zhang, J., Pollak, S., Haïssaguerre, M., et al. (2010). Early repolarization associated with sudden death: Insights from noninvasive electrocardiographic imaging. Heart Rhythm, 534-537.

33. Gima, K., \& Rudy, Y. (2002). Ionic current basis of electrocardiographic waveforms; a model study. Circulation Research, 90, 889-896.

34. Grandi, E., Puglisi, J. L., Bers, D. M., \& Severi, S. (2006). Compound mutations in long QT syndrome assessed by a computer model. In Comp. in Cardiol., Valencia.

35. Gulrajani, R. M., \& Mailloux, G. E. (1983). A simulation study of the effects of torso inhomogeneities on electrocardiographic potentials, using realistic heart and torso models. Circulation Research, 52, 45-56.

36. Hamlin, R. L., \& Scher, A. M. (1961). Ventricular activation process and genesis of QRS complex in the goat. The American Journal of Physiology, 200, 223-228.

37. Han, C., Pogwizd, S. M., Killingsworth, C. R., \& He, B. (2011). Noninvasive imaging of three-dimensional cardiac activation sequence during pacing and ventricular tachycardia. Heart Rhythm, 8, 1266-1272. doi:10.1016/j.hrthm. 2011.03.014.

38. Henriquez, C. S., Muzikant, A. L., \& Smoak, C. K. (1996). Anisotropy, fiber curvature, and bath loading effects on activation in thin and thick cardiac tissue preparations: Simulations in a three-dimensional bidomain model. Journal of Cardiovascular Electrophysiology, 7, 424-444.

39. Hodgkin, A. L., \& Huxley, A. F. (1952). A quantitative description of membrane current and its application to conduction and excitation in nerve. Journal of Physiology, 117, $500-544$.

40. Hoogendijk, M. G., Potse, M., Linnenbank, A. C., Verkerk, A. O., den Ruijter, H. M., van Amersfoorth, S. C. M., et al. (2010). Mechanism of right precordial ST-segment elevation in structural heart disease: Excitation failure by current-toload mismatch. Heart Rhythm, 7, 238-248.

41. Hoogendijk, M. G., Potse, M., Vinet, A., de Bakker, J. M. T., \& Coronel, R. (2011). ST-segment elevation by current-toload mismatch: An experimental and computational study. Heart Rhythm, 8, 111-118. doi:10.1016/j.hrthm.2010.09.066.

42. Hopenfeld, B., Stinstra, J. G., \& MacLeod, R. S. (2004). Mechanism for ST depression associated with contiguous subendocardial ischemia. Journal of Cardiovascular Electrophysiology, 15(10), 1200-1206.

43. Huygens, C. (1690). Treatise on light (English translation by S. P. Thompson, 1912). Chicago: University of Chicago Press.

44. Iyer, V., Mazhari, R., \& Winslow, R. L. (2004). A computational model of the human left-ventricular epicardial myocyte. Biophysical Journal, 87, 1507-1525.

45. Jacquemet, V., \& Henriquez, C. S. (2009). Genesis of complex fractionated atrial electrograms in zones of slow conduction: A computer model of microfibrosis. Heart Rhythm, 6, 803-810.

46. Jia, P., Ramanathan, C., Ghanem, R. N., Ryu, K., Varma, N., \& Rudy, Y. (2006). Electrocardiographic imaging of cardiac resynchronization therapy in heart failure: Observation of variable electrophysiologic responses. Heart Rhythm, 3, 296-310.

47. Johnston, P. R., \& Kilpatrick, D. (2003). The effect of conductivity values on ST segment shift in subendocardial ischaemia. IEEE Transactions on Biomedical Engineering, $50(2), 150-158$.

48. Keener, J. P. (1986). A geometrical theory for spiral waves in excitable media. SIAM Journal on Applied Mathematics, 46, 1039-1056.
49. Keener, J. P. (1991). An eikonal-curvature equation for action potential propagation in myocardium. Journal of Mathematical Biology, 29,629-651.

50. Keldermann, R. H., Nash, M. P., Gelderblom, H., Wang, V. Y., \& Panfilov, A. V. (2010). Electromechanical wavebreak in a model of the human left ventricle. American Journal of Physiology. Heart and Circulatory Physiology, 299, H134-143.

51. Keller, D. U. J., Seemann, G., Weiss, D. L., Farina, D., Zehelein, J., \& Dössel, O. (2007). Computer based modeling of the congenital long-QT 2 syndrome in the visible man torso: From genes to ECG. In 29th annu. int. conf. IEEE EMBS (pp. 1410-1413). Lyon, France.

52. Keller, D. U. J., Jarrousse, O., Fritz, T., Ley, S., Dössel, O., Seemann, G. (2011). Impact of physiological ventricular deformation on the morphology of the T-wave: A hybrid, static-dynamic approach. IEEE Transactions on Biomedical Engineering, 58, 2109-2119.

53. Kerckhoffs, R. C. P., Faris, O. P., Bovendeerd, P. H. M., Prinzen, F. W., Smits, K., McVeigh, E. R., et al. (2003). Timing of depolarization and contraction in the paced canine left ventricle: Model and experiment. Journal of Cardiovascular Electrophysiology, 14 Suppl., S188-S195.

54. Kerckhoffs, R. C. P., Lumens, J., Vernooy, K., Omens, J. H., Mulligan, L. J., Delhaas, T., et al. (2008). Cardiac resynchronization: Insight from experimental and computational models. Progress in Biophysics and Molecular Biology, 97, 543-561.

55. Kuijpers, N. H. L., ten Eikelder, H. M. M., Bovendeerd, P. H. M., Verheule, S., Arts, T., Hilbers, P. A. J. (2008). Mechanoelectric feedback as a trigger mechanism for cardiac electrical remodeling: A model study. Annals of Biomedical Engineering, 36, 1816-1835.

56. Kuijpers, N. H. L., Hermeling, E., Bovendeerd, P. H. M., Delhaas, T., \& Prinzen, F. W. (2012). Mechano-electrical coupling in dyssynchronous and failing hearts. J. Cardiovasc Trans Res, 5. doi:10.1007/s12265-012-9346-y.

57. Kurata, Y., Hisatome, I., Matsuda, H., \& Shibamoto, T. (2005). Dynamical mechanisms of pacemaker generation in $I_{\mathrm{K} 1}$-downregulated human ventricular myocytes: Insights from bifurcation analyses of a mathematical model. Biophysical Journal, 89, 2865-2887.

58. Lines, G. T., Buist, M. L., Grøttum, P., Pullan, A. J., Sundnes, J., \& Tveito, A. (2003). Mathematical models and numerical methods for the forward problem in cardiac electrophysiology. Computing and Visualization in Science, 5, 215-239.

59. Lorange, M., \& Gulrajani, R. M. (1986). Computer simulation of the Wolff-Parkinson-White preexcitation syndrome with a modified Miller-Geselowitz heart model. IEEE Transactions on Biomedical Engineering, 33(9), 862873.

60. Lorange, M., \& Gulrajani, R.M. (1993). A computer heart model incorporating anisotropic propagation: I. Model construction and simulation of normal activation. Journal of Electrocardiology, 26(4), 245-261.

61. Lorange, M., Gulrajani, R. M., Nadeau, R. A., \& Préda, I. (1993). A computer heart model incorporating anisotropic propagation: II. Simulations of conduction block. Journal of Electrocardiology, 26(4), 263-277.

62. MacLeod, R. S., \& Brooks, D. H. (1998). Recent progress in inverse problems in electrocardiology. IEEE Engineering in Medicine and Biology Magazine, 17(1), 73-83.

63. MacLeod, R. S., Gardner, M., Miller, R. M., \& Horáček, B. M. (1995). Application of an electrocardiographic inverse solution to localize ischemia during coronary angio- 
plasty. Journal of Cardiovascular Electrophysiology, 6(1), 2-18.

64. Miller, W. T. III., \& Geselowitz, D. B. (1978). Simulation studies of the electrocardiogram; I. The normal heart. Circulation Research, 43(2), 301-315.

65. Miri, R., Graf, I. M., \& Dössel, O. (2009). Efficiency of timing delays and electrode positions in optimization of biventricular pacing: A simulation study. IEEE Transactions on Biomedical Engineering, 56(11), 2573-2582.

66. Modre, R., Tilg, B., Fischer, G., \& Wach, P. (2002). Noninvasive myocardial activation time imaging: A novel inverse algorithm applied to clinical ECG mapping data. IEEE Transactions on Biomedical Engineering, 49, 1153-1161.

67. Modre, R., Seger, M., Fischer, G., Hintermüller, C., Hayn, D., Pfeifer, B., et al. (2006). Cardiac anisotropy: Is it negligible regarding noninvasive activation time imaging? IEEE Transactions on Biomedical Engineering, 53(4), 569-580.

68. Moe, G. K., Rheinboldt, W. C., \& Abildskov, J. A. (1964). A computer model of atrial fibrillation. American Heart Journal, 67, 200-220.

69. Muzikant, A. L., \& Henriquez, C. S. (1998). Bipolar stimulation of a three-dimensional bidomain incorporating rotational anisotropy. IEEE Transactions on Biomedical Engineering, 45(4), 449-462.

70. Nasmith, J. B., Pharand, C., Dubé, B., Matteau, S., LeBlanc, A. R., \& Nadeau, R. (2001). Localization of maximal ST segment displacement in various ischemic settings by orthogonal ECG: Implications for lead selection and the mechanism of ST shift. Canadian Journal of Cardiology, 17(1), 57-62.

71. Niederer, S., Mitchell, L., Smith, N., \& Plank, G. (2011). Simulating a human heart beat with near-real time performance. Frontiers in Physiology, 2, 14. doi:10.3389/fphys. 2011.00014.

72. Niimi, N., Sugiyama, S., Wada, M., Sugenoya, J., Oguri, H., Toyama, J., et al. (1977). Genesis of body surface potential distribution in right bundle branch block. Journal of Electrocardiology, 10, 257-266.

73. Noble, D., \& Rudy, Y. (2001). Models of cardiac ventricular action potentials: Iterative interaction between experiment and simulation. Philosophical Transactions of the Royal Society A-Mathematical, Physical, and Engineering Sciences, 359, 1127-1142.

74. Nygren, A., Fiset, C., Firek, L., Clark, J. W., Lindblad, D. S., Clark, R. B., et al. (1998). Mathematical model of an adult human atrial cell; the role of $\mathrm{K}^{+}$currents in repolarization. Circulation Research, 82, 63-81.

75. O' Cannon, R. III. (1985). Ischemia at a distance-so close yet so far. Journal of the American College of Cardiology, 6(1), 46-48.

76. Okajima, M., Fujino, T., Kobayashi, T., \& Yamada, K. (1968). Computer simulation of the propagation process in excitation of the ventricles. Circulation Research, 23, 203211.

77. van Oosterom, A., \& Oostendorp, T. F. (2004). ECGSIM: An interactive tool for simulating QRST waveforms. Heart, 90, 165-168.

78. van Oosterom, A., \& Plonsey, R. (1991). The Brody effect revisited. Journal of Electrocardiology, 24(4), 339-348.

79. Plonsey, R. (1979). A contemporary view of the ventricular gradient of Wilson. Journal of Electrocardiology, 12, 337341.

80. Potse, M., Dubé, B., Richer, J., Vinet, A., \& Gulrajani, R. M. (2006). A comparison of monodomain and bidomain reaction-diffusion models for action potential propagation in the human heart. IEEE Transactions on Biomedical Engineering, 53(12), 2425-2435. doi:10.1109/TBME.2006.880875.
81. Potse, M., Coronel, R., Falcao, S., LeBlanc, A.R., \& Vinet, A. (2007). The effect of lesion size and tissue remodeling on ST deviation in partial-thickness ischemia. Heart Rhythm 4(2), 200-206.

82. Potse, M., Dubé, B., Vinet, A. (2009). Cardiac anisotropy in boundary-element models for the electrocardiogram. Medical \& Biological Engineering \& Computing, 47, 719729. doi:10.1007/s11517-009-0472-x.

83. Potse, M., Vinet, A., Opthof, T., Coronel, R. (2009). Validation of a simple model for the morphology of the $\mathrm{T}$ wave in unipolar electrograms. American Journal of Physiology. Heart and Circulatory Physiology, 297, H792801. doi:10.1152/ajpheart.00064.2009.

84. Puglisi, J., Bers, D. M. (2001). LabHEART: An interactive computer model of rabbit ventricular myocyte ion channels and Ca transport. American Journal of Physiology. Cell Physiology, 281, C2049-2060.

85. Pullan, A. J., Cheng, L. K., Nash, M. P., Bradley, C. P., \& Paterson, D. J. (2001). Noninvasive electrical imaging of the heart: Theory and model development. Annals of Biomedical Engineering, 29, 817-836.

86. Qu, F., Li, L., Nikolski, V. P., Sharma, V., \& Efimov, I. R. (2005). Mechanisms of superiority of ascending ramp waveforms: New insights into mechanisms of shock-induced vulnerability and defibrillation. American Journal of Physiology. Heart and Circulatory Physiology, 289, H569577.

87. Reumann, M., Farina, D., Miri, R., Lurz, S., Osswald, B., \& Dössel, O. (2007). Computer model for the optimization of AV and VV delay in cardiac resynchronization therapy. Medical \& Biological Engineering \& Computing, 45, 845854.

88. Reumann, M., Gurev, V., \& Rice, J. J. (2009). Computational modeling of cardiac disease: Potential for personalized medicine. Personalized Medicine, 6, 45-66.

89. Rodríguez, B., Tice, B. M, Eason, J. C., Aguel, F., Ferrero, J. M. Jr, \& Trayanova, N. (2004). Effect of acute global ischemia on the upper limit of vulnerability: A simulation study. American Journal of Physiology. Heart and Circulatory Physiology, 286, H2078-H2088.

90. Romero, D., Sebastian, R., Bijnens, B. H., Zimmerman, V., Boyle, P. M., Vigmond, E. J., et al. (2010). Effects of the Purkinje system and cardiac geometry on biventricular pacing: A model study. Annals of Biomedical Engineering, 38, 1388-1398.

91. Rosenbaum, M. B., Elizari, M. V., Levi, R. J., Nau, G. J., Pisani, N., Lázzari, J. O., et al. (1969). Five cases of intermittent left anterior hemiblock. American Journal of Cardiology, 24, 1-7.

92. Rudy, Y., Plonsey. R., Liebman. J. (1979). The effects of variations in conductivity and geometrical parameters on the electrocardiogram, using an eccentric spheres model. Circulation Research, 44, 104-111.

93. Salu, Y., Marcus, M. L. (1976). Computer simulation of the precordial QRS complex: Effects of simulated changes in ventricular wall thickness and volume. American Heart Journal, 92, 758-766.

94. Saucerman, J. J., Healy, S. N., Belik, M. E., Puglisi, J. L., McCulloch, A. D. (2004). Proarrhythmic consequences of a KCNQ1 AKAP-binding domain mutation; computational models of whole cells and heterogeneous tissue. Circulation Research, 95, 1216-1224.

95. Scacchi, S., Colli Franzone, P., Pavarino, L. F., Taccardi, B. (2009). A reliability analysis of cardiac repolarization time markers. Mathematical Biosciences, 219, 113128. 
96. Scher, A. M., \& Young, A. C. (1957) Ventricular depolarization and the genesis of QRS. Annals of the New York Academy of Sciences, 65, 768-778.

97. Schmitt, O. H. (1969). Biological information processing using the concept of interpenetrating domains. In: K. N. Leibovic (Ed.), Information processing in the nervous system (pp. 325-331). New York: Springer.

98. Selvester, R. H., Solomon, J. C., \& Gillespie, T. L. (1968). Digital computer model of a total body electrocardiographic surface map; an adult male-torso simulation with lungs. Circulation 38, 684-690.

99. Solomon, J. C., \& Selvester, R. H. (1973). Simulation of measured activation sequence in the human heart. American Heart Journal, 85, 518-523.

100. Spach, M. S., Barr, R. C., Serwer, G. A., Kootsey, J. M., \& Johnson, E. A. (1972). Extracellular potentials related to intracellular action potentials in the dog Purkinje system. Circulation Research, 30(5), 505-519.

101. Spach, M. S., Miller, W. T. III., Miller-Jones, E., Warren, R. B., \& Barr, R. C. (1979). Extracellular potentials related to intracellular action potentials during impulse conduction in anisotropic canine cardiac muscle. Circulation Research, 45, 188-204

102. Spach, M. S., Heidlage, J. F., Dolber, P. C., \& Barr, R. C. (2007). Mechanism of origin of conduction disturbances in aging human atrial bundles: Experimental and model study. Heart Rhythm, 4, 175-185.

103. Strauss, D. G., Selvester, R. H., \& Wagner, G. S. (2011) Defining left bundle branch block in the era of cardiac resynchronization therapy. American Journal of Cardiology, 107, 927-934.

104. Streeter, D. D. Jr., Spotnitz, H. M., Patel, D. P., Ross, J., \& Sonnenblick, E. H. (1969). Fiber orientation in the canine left ventricle during diastole and systole. Circulation Research, 24, 339-347.

105. Sweeney, M. O., \& Prinzen, F. W. (2006). A new paradigm for physiologic ventricular pacing. Journal of the American College of Cardiology, 47, 282-288.

106. Szilágyi, S., Szilágyi, L., \& Benyó, Z. (2011). A patient specific electro-mechanical model of the heart. Computer Methods and Programs in Biomedicine, 101, 183-200.

107. Taccardi, B. (1963). Distribution of heart potentials on the thoracic surface of normal human subjects. Circulation Research, 12, 341-352.

108. Taccardi, B., Macchi, E., Lux, R. L., Ershler, P. R., Spaggiari, S., Baruffi, S., et al. (1994). Effect of myocardial fiber direction on epicardial potentials. Circulation, 90, 3076-3090.

109. Taggart, P., Sutton, P. M. I., Opthof, T., Coronel, R., Trimlett, R., Pugsley, W., et al. (2001). Transmural repolarisation in the left ventricle in humans during normoxia and ischaemia. Cardiovascular Research, 50, 454-462.

110. Tranquillo, J. V., Franz, M. R., Knollmann, B. C., Henriquez, A. P., Taylor, D. A., \& Henriquez, C. S. (2004). Genesis of the monophasic action potential: Role of intersti- tial resistance and boundary gradients. American Journal of Physiology. Heart and Circulatory Physiology, 286, H13701381.

111. Trayanova, N., Plank, G., \& Rodríguez, B. (2006). What have we learned from mathematical models of defibrillation and postshock arrhythmogenesis? Application of bidomain simulations. Heart Rhythm, 3, 1232-1235.

112. Trayanova, N. A., Constantino, J., \& Gurev, V. (2011). Electromechanical models of the ventricles. American Journal of Physiology. Heart and Circulatory Physiology, 301, H279-286. doi:10.1152/ajpheart.00324.2011.

113. Trudel, M. C., Dubé, B., Potse, M., Gulrajani, R. M., Leon, L. J. (2004). Simulation of propagation in a membranebased computer heart model with parallel processing. IEEE Transactions on Biomedical Engineering, 51(8), 13191329.

114. Tung, L. (1978). A bi-domain model for describing ischemic myocardial D-C potentials. Ph.D. thesis, MIT, Cambridge, MA, USA.

115. ten Tusscher, K. H. W. J., \& Panfilov, A. V. (2006). Alternans and spiral breakup in a human ventricular tissue model. American Journal of Physiology. Heart and Circulatory Physiology, 291, H1088-H1100. doi:10.1152/ajpheart. 00109.2006.

116. ten Tusscher, K. H. W. J., Noble, D., Noble, P. J., \& Panfilov, A. V. (2004). A model for human ventricular tissue. American Journal of Physiology. Heart and Circulatory Physiology, 286, H1573-H1589.

117. Varma, N., Jia, P., Rudy, Y. (2007). Electrocardiographic imaging of patients with heart failure with left bundle branch block and response to cardiac resynchronization therapy. Journal of Electrocardiology, 40, S174-178.

118. Varma, N., Jia, C. P., Ramanathan, C., \& Rudy, Y. (2010). $\mathrm{RV}$ electrical activation in heart failure during right, left, and biventricular pacing. Journal of the American College of Cardiology: Cardiovascular Imaging, 3, 567-575.

119. Vigmond, E. J. (2005). The electrophysiological basis of MAP recordings. Cardiovascular Research, 68, 502-503 (letter).

120. Vigmond, E. J., \& Leon, L. J. (1999). Electrophysiological basis of mono-phasic action potential recordings. Medical \& Biological Engineering \& Computing, 37, 359-365.

121. Wehrens, X. H. T., Abriel, H., Cabo, C., Benhorin, J., \& Kass, R. S. (2000). Arrhythmogenic mechanism of an LQT-3 mutation of the human heart $\mathrm{Na}^{+}$channel $\alpha$-subunit; a computational analysis. Circulation, 102, 584-590.

122. Wikswo, J. P., Wisialowski, T. A., Altemeier, W. A., Balser, J. R., Kopelman, H. A., \& Roden, D. M. (1991). Virtual cathode effects during stimulation of cardiac muscle; twodimensional in vivo experiments. Circulation Research, 68, 513-530.

123. Williams, G. S. B., Smith, G. D., Sobie, E. A., \& Jafri, M. S. (2010). Models of cardiac excitation-contraction coupling in ventricular myocytes. Mathematical Biosciences, 226 1-15, (review). 